\title{
Time-gated transillumination of biological tissues and tissuelike phantoms
}

\author{
Gerhard Mitic, Jochen Kölzer, Johann Otto, Erich Plies, Gerald Sölkner, and \\ Wolfgang Zinth
}

\begin{abstract}
The applicability and limits of time-resolved transillumination to determine the internal details of biological tissues are investigated by phantom experiments. By means of line scans across a sharp edge, the spatial resolution $(\Delta x)$ and its dependence on the time-gate width $(\Delta t)$ can be determined. Additionally, measurements of completely absorbing bead pairs embedded in a turbid medium demonstrate the physical resolution in a more realistic case. The benefit of time resolution is especially high for a turbid medium with a comparatively small reduced scattering coefficient of approximately $\mu_{s}{ }^{\prime}=0.12$ $\mathrm{mm}^{-1}$. Investigations with partially absorbing beads and filled plastic tubes demonstrate the high sensitivity of time-resolving techniques with respect to spatial variations in scattering or absorption coefficients that are due to the embedded disturber. In particular, it is shown that time gating is sensitive to variations in scattering coefficients.
\end{abstract}

Key words: Time-resolved transillumination, turbid media, light scattering, streak camera.

\section{Introduction}

The noninvasive diagnosis of tissue by light is of great interest for the examination of organs as well as for preventive or other medical routines. ${ }^{1,2}$ Of particular interest in this area is the transillumination of the female breast for a preventive checkup. For detecting breast cancer at an early stage, transillumination with light may be considered a promising alternative to potentially harmful x-ray mammography. Because of the enormous multiple scattering of light in the tissue, however, this approach suffers from restricted spatial resolution. But the separation of multiply scattered photons with long path lengths from those with short path lengths enables an improvement in spatial resolution to be achieved by means of time resolution. Chance et al..$^{3,4}$ and Delpy et $a l .{ }^{5}$ have introduced the time-resolved technique to near-infrared spectroscopy. Several different time-

G. Mitic, J. Kölzer, J. Otto, and G. Sölkner are with Siemens AG, ZFE ST KM 63, Otto-Hahn-Ring 6, D-81739 Munich, Germany; E. Plies is with the Institut für Angewandte Physik, Universität Tübingen, Auf der Morgenstelle 10, D-72076 Tübingen, Germany; W. Zinth is with the Institut für Medizinische Optik, LudwigMaximilians-Universität, Barbarastrasse 16, D-80797 Munich, Germany.

Received 5 October 1993; revised manuscript received 18 February 1994.

0003-6935/94/286699-12\$06.00/0.

(C) 1994 Optical Society of America. domain or frequency-domain methods have been realized for imaging through highly scattering media. In the frequency domain, phase-resolved methods that use intensity-modulated light have been investigated by different authors. ${ }^{6-9}$ In the time domain, the improvement in spatial resolution is achieved by means of a time-of-flight restriction of scattered photons. ${ }^{10}$ The transmitted light can be measured by the use of a streak camera ${ }^{11}$ or by a fast microchannel plate photomultiplier tube. ${ }^{12}$ The presented timedomain experiments were performed with a streak camera. ${ }^{13}$

Several authors have published time-resolved in vitro experiments at a specimen thickness of several centimeters. ${ }^{14,15}$ In spite of these measurements, the benefit of time gating for realistic samples was not investigated systematically. Published time-resolved experiments often refer to an unrealistic specimen thickness of less than $10 \mathrm{~mm}$ or have been performed on turbid media with optical properties that do not correspond to biological tissue. In addition the optical tissue parameters (reduced scattering coefficient $\mu_{s}{ }^{\prime}$, absorption coefficient $\mu_{A}$ ) depend not only on the type of tissue but also on the optical wavelength used. ${ }^{16}$ Until now, there have been hardly any publications presenting time-resolved in vivo experiments of the female breast.

Therefore in this paper we present in vivo experiments that we performed on volunteers by using a Ti:sapphire laser to determine the optical properties 
of the human female breast at the wavelength of the isosbestic point of oxyhemoglobin and deoxyhemoglo$\operatorname{bin}(\lambda=800 \mathrm{~nm})$.

Further measurements were carried out on different tissue-equivalent phantoms (turbid media with inserted absorbers) to clarify the benefits offered by a time-resolved transillumination technique in quantitative terms. The benefit of time gating was investigated for a wide range of optical properties of both the surrounding turbid medium and the embedded inhomogeneity. In this way measurements on an edge embedded in a turbid medium and on phantoms with completely and partially absorbing objects of different shapes were examined.

\section{Experimental Setup}

The experimental setup for time-resolved light transillumination ${ }^{10}$ can be seen from Fig. 1. The laser system for the phantom experiments consists of a mode-locked Nd:YAG laser (repetition frequency 82 $\mathrm{MHz}$, pulse width $100 \mathrm{ps}$ ) with subsequent pulse compression (7 ps) and frequency doubling $(\lambda=532$ $\mathrm{nm})$. Time-resolved in vivo experiments were performed with a mode-locked Ti:sapphire laser (82 $\mathrm{MHz}, 80 \mathrm{fs}$ ) at a wavelength of $\lambda=800 \mathrm{~nm}$.

A beam for triggering the synchroscan streak camera (S1 photocathode, Hamamatsu C3681) and a reference beam are derived from the main beam. Unlike the probe beam, the reference beam does not traverse the phantom to be examined but is incident upon the streak camera slit on the detection side to determine the temporal zero. Light from the probe beam is scattered in the phantom and finally reaches the detection side after many scattering events. This diffuse light from the detection side of the phantom is imaged onto the slit of the streak camera with a 1:1 magnification. The slit has a dimension of $50 \mu \mathrm{m} \times 6 \mathrm{~mm}$, and the numerical aperture of the streak-camera optic is 0.22 . The streak camera records the temporal profile of the incident light intensity and displays it as a spatial profile (time resolution $10 \mathrm{ps}$ ). The phantom is located on an $x-y$ stage and can be moved in the horizontal plane under computer control.

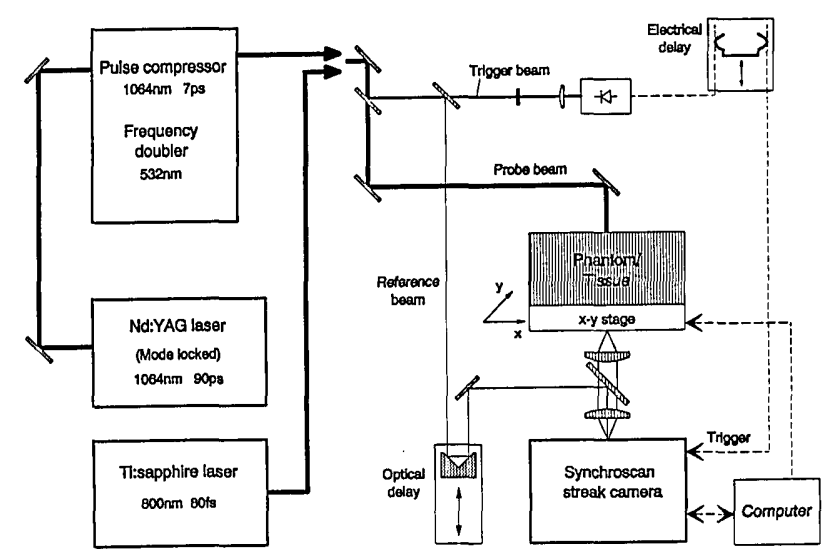

Fig. 1. Schematic setup for time-resolved transillumination of turbid media.
Milk was used as the turbid medium (whole-milk powder from Töpfer, D-87463 Dietmannsried/Allgäu, Germany), and the optical parameters were set by the dilution of the milk and by the addition of ink (Encre Noire from Waterman, France). To obtain a reduced scattering coefficient of $\mu_{s}{ }^{\prime}=4.5 \mathrm{~mm}^{-1}$ at a wavelength of $\lambda=532 \mathrm{~nm}$, a quantity of 230 -g milk powder has to be stirred into 1.5-L water (see Section 3). If the milk is diluted, the reduced scattering coefficient cannot simply be calculated with a linear relation because of the modified consistency of the milk. At high concentrations the fat droplets in the emulsion are not independent of each other and therefore the Mie-scattering function is changed if the milk is diluted. As a consequence $\mu_{s}^{\prime}=0.9 \mathrm{~mm}^{-1}$ is obtained when only $30-\mathrm{g}$ milk powder is dissolved in 1.5-L water.

The experimental setup was tested with different scattering media in order to control the linearity of the intensity and of the time scale. For each phantom a line scan was performed in the 500-ps full range of the streak camera to evaluate time gates from 15 to $250 \mathrm{ps}$ and in the 2-ns full range to evaluate time gates from 250 to $\sim 1500 \mathrm{ps}$. The continuous-wave (cw) case was determined from a third line scan with the streak camera in the static mode. The intensity of the probe beam was adjusted according to the optical properties of the turbid medium. For a phantom with a reduced scattering coefficient of $\mu_{s}{ }^{\prime} \approx 1 \mathrm{~mm}^{-1}$ and a thickness of $40 \mathrm{~mm}$, the intensity was $\sim 100 \mathrm{~mW}$, and a measuring time for each scan point of $\sim 10 \mathrm{~s}$ was required for obtaining a good signal-to-noise ratio.

To record a complete dispersion curve with a total temporal width of $\sim 6 \mathrm{~ns}$, several 2-ns measurements were combined. This was accomplished by the delay of the trigger signal for $1.75 \mathrm{~ns}$ by the use of an electrical delay unit. The correct overlap of each 2-ns segment was controlled by reference pulses in regular intervals of $\sim 1.75 \mathrm{~ns}$. To obtain the correct dispersion curve, a precise shading correction and dark subtraction was performed for each measurement.

\section{Determination of Scattering and Absorption Coefficients}

For all quantitative experiments it is necessary to deduce the optical properties of the turbid medium from the temporal dispersion curve. The diffusion model was used to determine the absorption coefficient $\mu_{A}$ and the reduced scattering coefficient $\mu_{s}{ }^{\prime}=$ $\mu_{s}(1-g)$ ( $g$ is the anistropy factor of scattering). $A$ solution of the diffusion equation for a homogeneous medium was used according to Eq. (14) in Patterson et al. ${ }^{17}$ It turned out that the diffusion model permits a good description of light propagation in turbid media with a specimen thickness of $40 \mathrm{~mm}$ as long as the reduced scattering coefficient was greater than $0.5 \mathrm{~mm}$, i.e., as long as many scattering events occurred within the specimen. When the calculated temporal dispersion curve is fit to the 
measured curve, both the reduced scattering coefficient $\mu_{s}{ }^{\prime}$ and the absorption coefficient $\mu_{A}$ can be determined. ${ }^{18}$ To examine this approach, theoretical curves were fitted to the measured dispersion curves (displayed normalized) for phantoms consisting of milk with two different concentrations of ink and having a thickness of $40 \mathrm{~mm}$ (Fig. 2). Time zero is given by the incidence of the laser pulse upon the scattering medium. Excellent agreement between the measured and the calculated curves was found.

To obtain an estimate for the optical properties of the female breast at the wavelength close to the isosbestic point of oxyhemoglobin and deoxyhemoglobin, in vivo measurements with a Ti:sapphire laser $(\lambda=800 \mathrm{~nm})$ were performed. The mamma was slightly compressed to ensure a constant thickness. To avoid a changed blood perfusion, the compression was much weaker than in conventional mammography. The probe beam with a total power of 100-150 $\mathrm{mW}$ was expanded to a diameter of $10 \mathrm{~mm}$ to keep the power density below the maximum permissible exposure of $2 \mathrm{~mW} / \mathrm{mm}^{2}$ (see Ref. 19), which corresponds to the solar constant. This power density causes only a negligible heating of the skin of the mamma. Additionally, a beam blocker permitted the volunteers to interrupt the probe beam during the measurement. Besides this, the identical experimental setup was used (Fig. 1).

Measurements on compressed mammae were carried out with different volunteers of between 25 and 43 years of age. Figure 3 shows measured dispersion curves for three volunteers and the corresponding theoretical curves calculated according to the diffusion model. The dispersion curves range over a period of $\sim 6 \mathrm{~ns}$ with a mean time of flight of more than 2 ns. This means that most photons travel the tenfold geometric distance between entry and exit of the mamma because of the immense scattering and the relatively weak absorption of the tissue. For volunteers 1 and $2(D=45 \mathrm{~mm})$, the signal overcomes

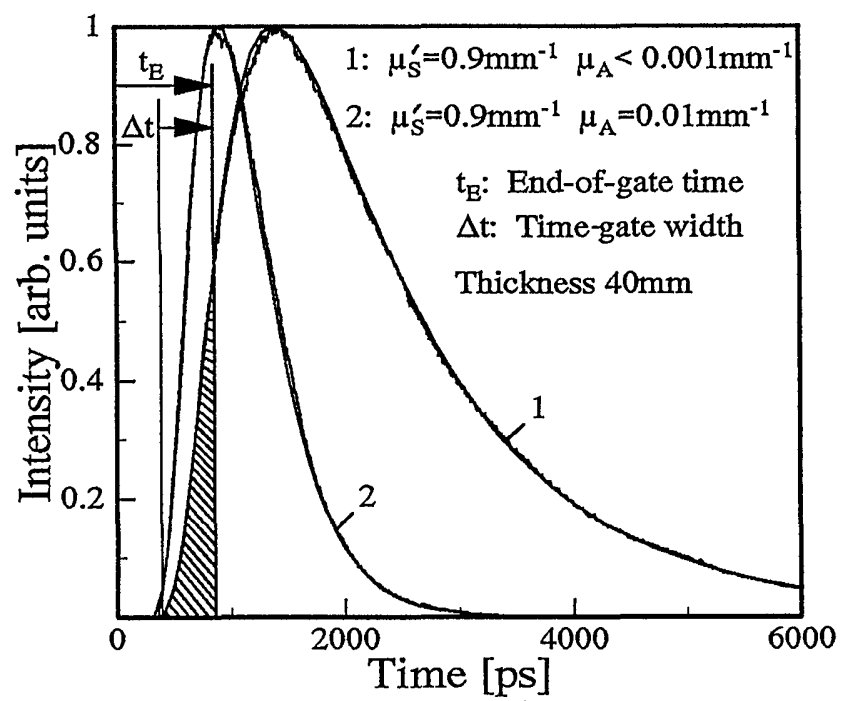

Fig. 2. Measured dispersion curves on phantom and associated theoretical fit curves.

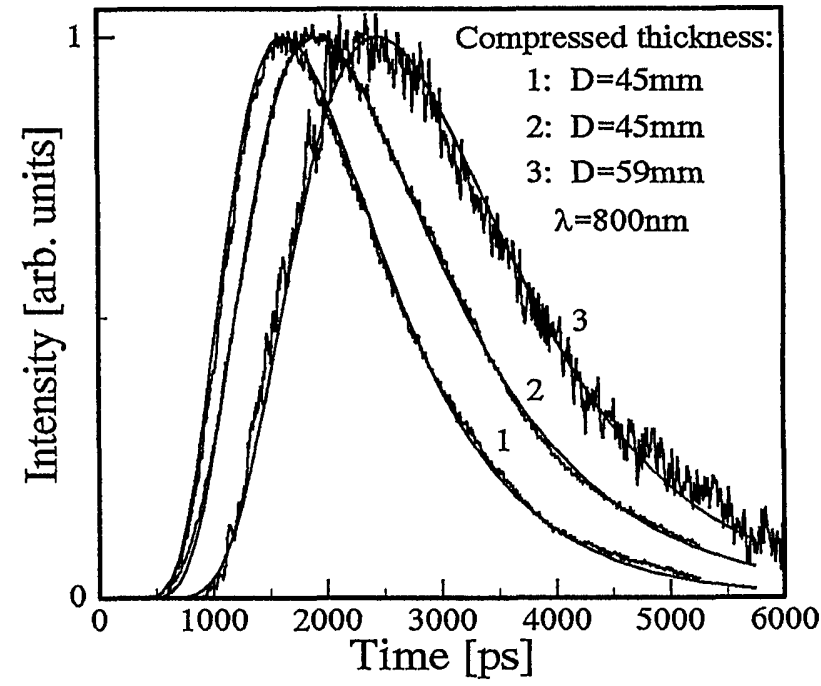

Fig. 3. In vivo dispersion curves of the compressed mamma (thickness $D$ ) and corresponding theoretical fit curves for three volunteers.

the background noise after a time of flight of $510 \mathrm{ps,}$ which is more than twice the minimum time of flight of a photon without scattering. For volunteer 3 $(D=59 \mathrm{~mm})$, this time shifts to $830 \mathrm{ps}$, which is approximately three times as much as the minimum time of flight (refractive index 1.4). ${ }^{17}$ The stronger noise on signal for curve 3 is due to the greater thickness of the compressed mamma of volunteer 3 compared with volunteers 1 and 2 . The corresponding optical parameters of the fit curves can be seen in Table 1. In summary, reduced scattering coefficients ranging from $\mu_{s}{ }^{\prime}=0.72 \mathrm{~mm}^{-1}$ to $\mu_{s}{ }^{\prime}=1.22$ $\mathrm{mm}^{-1}$ and corresponding absorption coefficients from $\mu_{A}=0.0017 \mathrm{~mm}^{-1}$ to $\mu_{A}=0.0032 \mathrm{~mm}^{-1}$ were measured for human breast tissue in vivo at a

Table 1. Reduced Scattering and Absorption Coefficients for Mammary Tissue In Vivo at $800 \mathrm{~nm}$ Compared with Milk at 532 and $800 \mathrm{~nm}$

\begin{tabular}{|c|c|c|c|c|c|}
\hline \multirow{2}{*}{$\begin{array}{c}\text { Mamma } \\
\text { In Vivo/ } \\
\text { Turbid Medium }\end{array}$} & \multirow[b]{2}{*}{$\begin{array}{l}\text { Age } \\
\text { (yrs) }\end{array}$} & \multicolumn{2}{|c|}{$532 \mathrm{~nm}$ (Green) } & \multicolumn{2}{|c|}{$800 \mathrm{~nm}$ (Near Infrared) } \\
\hline & & $\begin{array}{c}\mu_{s}^{\prime} \\
\left(\mathrm{mm}^{-1}\right)\end{array}$ & $\begin{array}{c}\mu_{A} \\
\left(\mathrm{~mm}^{-1}\right)\end{array}$ & $\begin{array}{c}\mu_{s}^{\prime} \\
\left(\mathrm{mm}^{-1}\right)\end{array}$ & $\begin{array}{c}\mu_{A} \\
\left(\mathrm{~mm}^{-1}\right)\end{array}$ \\
\hline $\begin{array}{l}\text { Volunteer } 1 \\
\text { (Fig. 3) }\end{array}$ & 39 & & & 1.09 & 0.0032 \\
\hline $\begin{array}{l}\text { Volunteer } 2 \\
\text { (Fig. 3) }\end{array}$ & 34 & & & 1.22 & 0.0022 \\
\hline $\begin{array}{l}\text { Volunteer } 3 \\
\text { (Fig. 3) }\end{array}$ & 43 & & & 1.05 & 0.0031 \\
\hline $\begin{array}{l}\text { Volunteer } 4 \\
\quad \text { (not shown) }\end{array}$ & 26 & & & 1.10 & 0.0028 \\
\hline $\begin{array}{l}\text { Volunteer } 5 \\
\quad \text { (not shown) }\end{array}$ & 42 & & & 0.72 & 0.0017 \\
\hline $\begin{array}{l}\text { Volunteer } 6 \\
\text { (not shown) }\end{array}$ & 27 & & & 1.00 & 0.0019 \\
\hline $\begin{array}{l}\text { Mamma in vivo } \\
\text { (Total range) }\end{array}$ & & & & $0.72-1.22$ & $017-0.0032$ \\
\hline Whole milk & & 4.5 & 0.001 & 2.5 & 0.0024 \\
\hline $\begin{array}{l}\text { 1/8 Diluted } \\
\text { whole milk }\end{array}$ & & 0.87 & $<0.001$ & & \\
\hline
\end{tabular}


wavelength of $800 \mathrm{~nm}$. Because breast tissue is inhomogeneous, consisting of fatty, glandular, and fibrocystic tissue, only a mean value for the optical properties of the mamma could be determined. Nonideal geometric conditions related to the compression of the mamma may be a source of error. However, repetition of the measurement after a short break gave consistent results within a maximum temporal shift of $\sim 100$ ps. Even good agreement between the dispersion curves of the right and the left mammae was found. For the present experimental setup it is impossible to determine the anisotropy factor $g$ directly, and therefore the scattering coefficient $\mu_{s}$ could not be calculated. The use of $g$ values from the literature does not solve the problem, as published $g$ values for different types of human breast tissue vary strongly. Values are given from $g=0.92$ for fibroglandular tissue and $g=0.95$ for adipose tissue to $g=0.88$ for $\operatorname{carcinomas}^{20}(\lambda=700 \mathrm{~nm})$, whereas Peters et al. specify $g$ for all types of tissue in the range $0.945-0.985$ as being invariant with wavelength. ${ }^{21}$ The use of these strongly varying $g$ values yields strong variations of the scattering coefficient $\mu_{s}=\mu_{s}{ }^{\prime} /(1-g)$ so that a determination of $\mu_{s}$ alone is of limited value. As a consequence, the optical parameters were fitted only with respect to the reduced scattering coefficient $\mu_{s}^{\prime}$ and the absorption coefficient $\mu_{A}$ for all phantom experiments.

Table 1 compares the optical properties of the human breast and milk for two wavelengths. The phantom experiments were carried out at a wavelength of $532 \mathrm{~nm}$ because of the simple and easy handling of this experimental setup. The in vivo experiments were performed at $800 \mathrm{~nm}$. This wavelength range is of special interest for medical diagnosis, as this is the isosbestic point of oxyhemoglobin and deoxyhemoglobin, and a variation of the wavelength that correlates with a change of $\mu_{A}$ yields specific diagnostic information. To simulate the in vivo tissue properties reasonably well by milk at $\lambda=$ $532 \mathrm{~nm}$, the optical properties of milk at this wave-

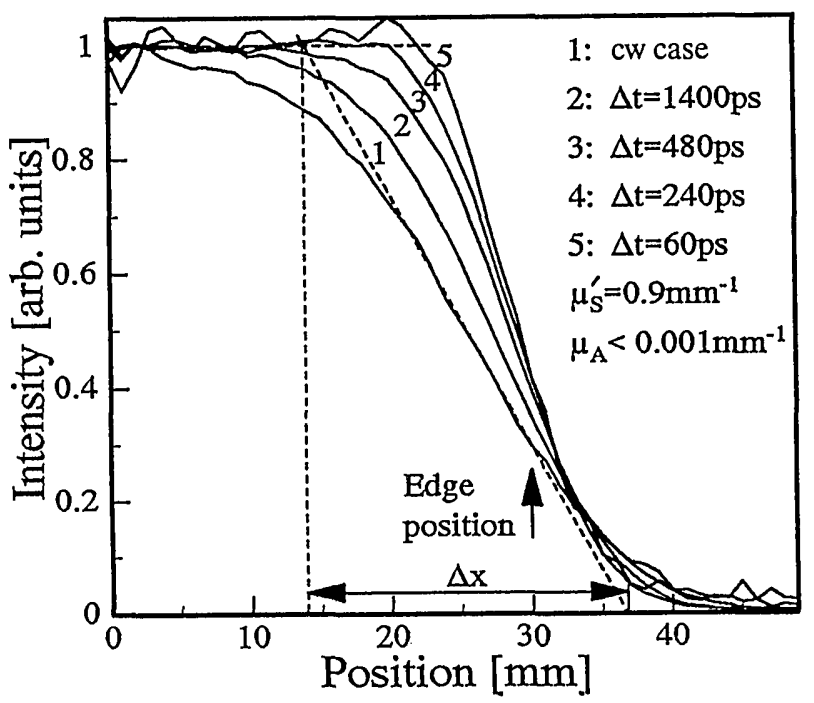

Fig. 4. Edge-spread functions for different time gates.
End-of-gate time $t_{\mathrm{E}}$ [ps]

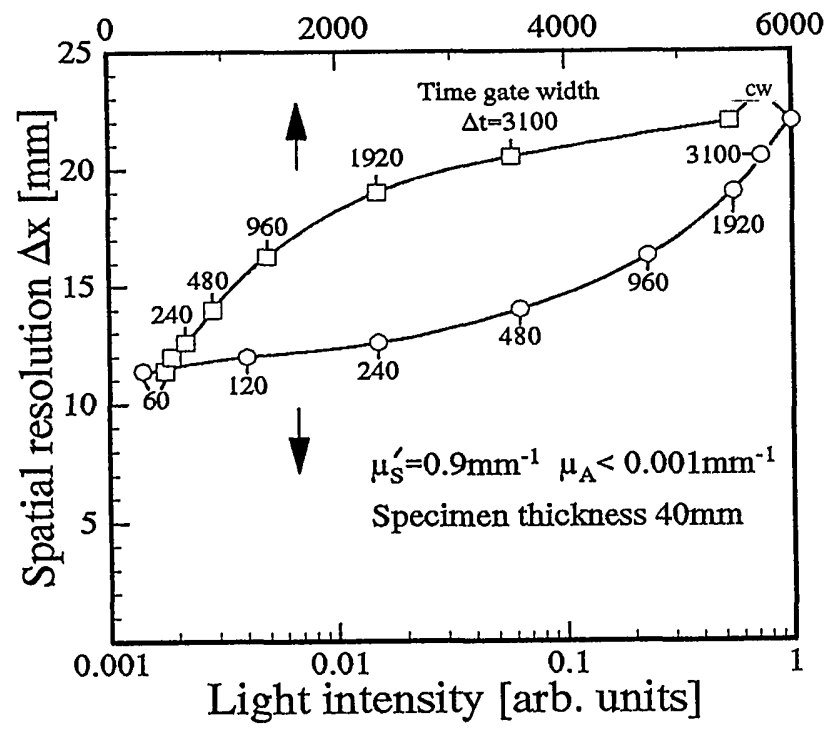

Fig. 5. Spatial resolution versus end-of-gate time and light intensity (the measurement symbols represent different time gates). The squares correspond to the top horizontal scale and the circles correspond to the bottom scale.

length have to be adjusted to the optical properties of human breast tissue at a wavelength of $800 \mathrm{~nm}$. This can be achieved by the dilution of the milk and the addition of ink. When one part of whole milk was diluted with seven parts of water, the reduced scattering coefficient increased to a breast tissuelike value of $0.9 \mathrm{~mm}^{-1}$. Absorption was neglected in most of the phantom experiments, as signal traces recorded at short times with $\mu_{A} \approx 0.003 \mathrm{~mm}^{-1}$ (the greatest values found in the in vivo experiments) show no significant difference from those recorded with $\mu_{A}<0.001 \mathrm{~mm}^{-1}$.

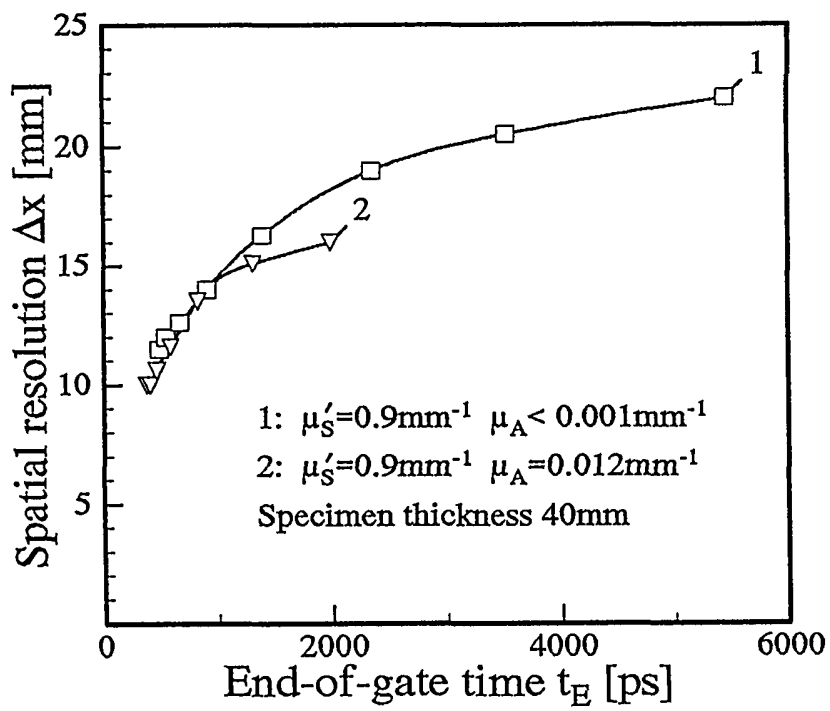

Fig, 6. Influence of an increase in absorption on the achievable improvement in spatial resolution by time gating. 


\section{Phantom Experiments}

Systematic measurements on phantoms were performed to determine the improvements that were due to time-resolved detection. For the possible application to breast cancer detection, turbid media with a wide range of optical parameters were investigated, with special emphasis on the optical properties of human breast tissue determined above. In general, the scattering medium to be transilluminated had a thickness of $40 \mathrm{~mm}$. All embedded objects were placed in the central plane of the turbid medium, where the strongest reduction of the spatial resolution that is due to the scattering occurs. The influence of the reduced scattering and absorption coefficient of the turbid medium on the resolution in the time gating experiments is shown by means of the width of the measured edge-spread function (Subsection 4.A). The case of totally absorbing objects embedded in the highly scattering medium demonstrates the spatial resolution achievable by the time-resolved technique (Subsection 4.B). The detectability of spatial variations in reduced scattering and absorption coefficients is investigated in Subsection 4.C.

\section{A. Edge-Spread Function}

Quantitative statements about the spatial resolution available by time-resolved detection are obtained from line scans across a sharp edge placed in the central plane of a turbid medium. ${ }^{22,23}$ The optical parameters of the scattering medium selected for these experiments are $\mu_{s}{ }^{\prime}=0.9 \mathrm{~mm}^{-1}$ and $\mu_{A}<0.001$ $\mathrm{mm}^{-1}$. Figure 2 shows a temporally dispersed pulse associated with these optical parameters (curve 1) and defines an end-of-gate time $t_{E}$ and a time gate $\Delta t$. The end-of-gate time $t_{E}$ is given with respect to the incidence of the laser pulse into the scattering medium, whereas $\Delta t$ represents the width of the time gate from which the image information is derived. A photon without scattering would traverse a phantom with a thickness of $40 \mathrm{~mm}$ in a minimum time of flight of $187 \mathrm{ps}$ (refractive index 1.4). As the probability that a photon is not scattered is vanishingly small, detectable amounts of scattered light occur only after $350 \mathrm{ps}$, which is approximately twice the minimum time of flight (optical parameters $\mu_{s}{ }^{\prime}=0.9 \mathrm{~mm}^{-1}$ and $\left.\mu_{A}<0.001 \mathrm{~mm}\right)$. Consequently the time gate $\Delta t$ starts at $t=350 \mathrm{ps}$, at which the signal overcomes the background noise. This point depends on the actual optical parameters of the scattering medium.

Below, the normalized intensity profiles result from integration over the respective time gates. Edgespread functions represent the detected intensity profile for a fixed $\Delta t$ when a sharp edge is scanned in the central plane of the scattering medium. Figure 4 shows the edge-spread function for five different time gates, the edge being located at a position $x=30 \mathrm{~mm}$. At the position of the edge, the normalized edgespread function shows values of $\sim 0.3-0.4$, depending on the time gate. Because of immense scattering, most photons traverse the central plane in the turbid medium several times at different positions, and therefore the edge-spread function at the position of the edge is less than 0.5. With a decreasing time gate, this value increases because the respective photons are less scattered.

Finally, the width of the resulting edge-spread function is determined according to Fig. 4. The slope of the tangent is determined by a least-squares procedure, and the intersections with the horizontal saturation lines of the edge-spread function define the spatial resolution $\Delta x$. Because of the relatively poor signal-to-noise ratio at short integration times, the spatial resolution $\Delta x$ (line resolution) is defined as the full width of the edge-spread function and not the common distance between the $10 \%$ value and the $90 \%$ value.

Figure 5 shows appropriate $\Delta x$ values for various times gates $\Delta t$. The axis above shows the end-ofgate time $t_{E}$ measured with respect to the incidence of
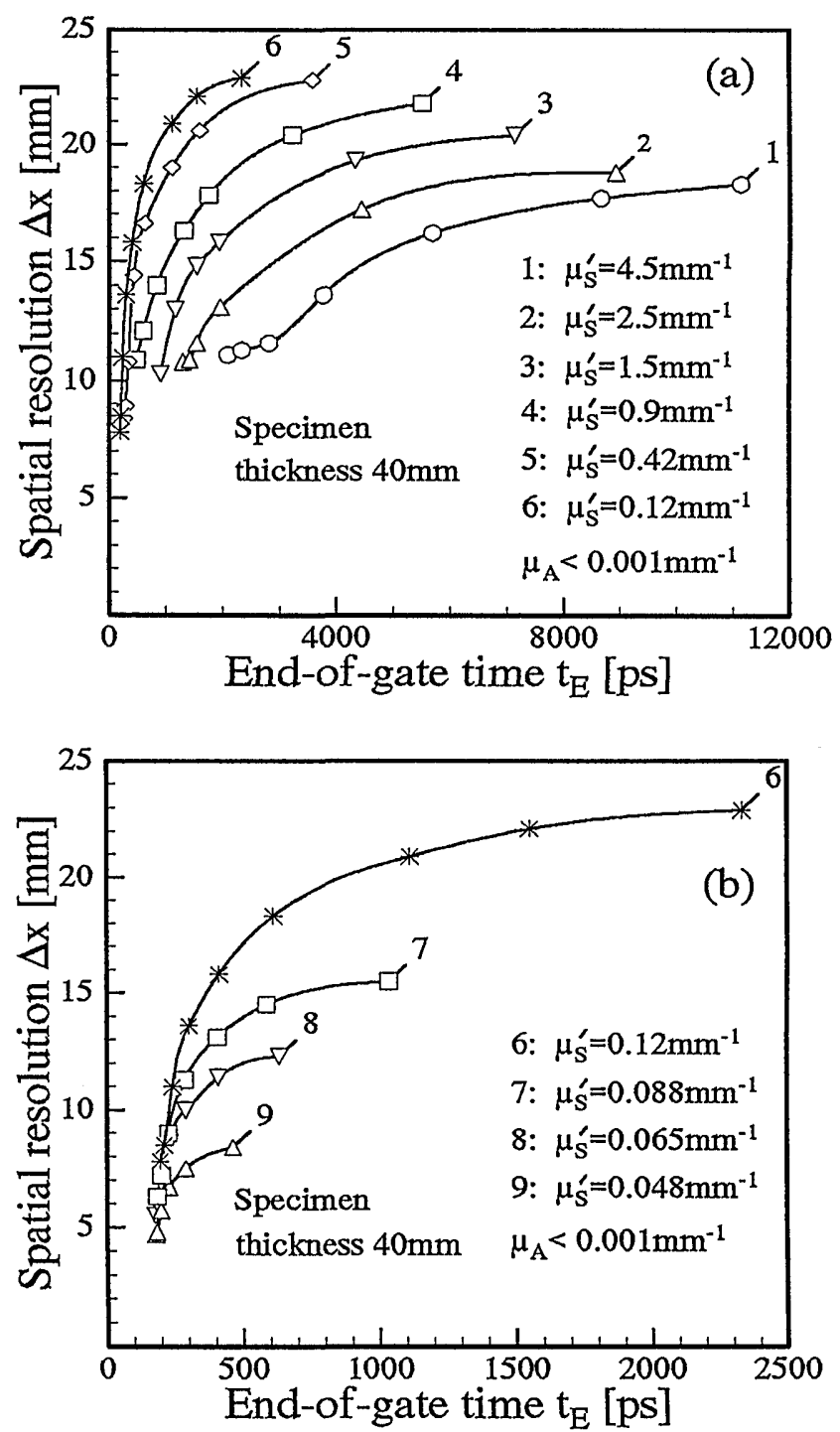

Fig. 7. Spatial resolution in relation to end-of-gate time for consecutively increasing reduced scattering coefficients (a) $\mu_{s}{ }^{\prime}=$ $0.12-4.5 \mathrm{~mm}^{-1},(\mathrm{~b}) \mu_{s}^{\prime}=0.048-0.12 \mathrm{~mm}^{-1}$. 
the laser pulse upon the scattering medium. For the cw case we used the width obtained by integrating the entire dispersion curve within the first $5 \mathrm{~ns}$ because this width was also attained for the cw measurement. The smallest time gate used was $\Delta t=60 \mathrm{ps}$. For the cw case a width of $22 \mathrm{~mm}$ was measured, whereas for $\Delta t=60 \mathrm{ps}$ the width was reduced to $11.5 \mathrm{~mm}$, i.e., under the present conditions $\left(\mu_{s}^{\prime}=0.9 \mathrm{~mm}^{-1}\right.$, $\mu_{A}<0.001 \mathrm{~mm}^{-1}$ ) the maximum gain factor of spatial resolution attainable by time resolution is 1.9 for a time gate of $60 \mathrm{ps}$. Reducing the time gate still further mainly increases the noise. The intensity loss that is due to time gating is seen if the spatial resolution is plotted as a function of the light inten-
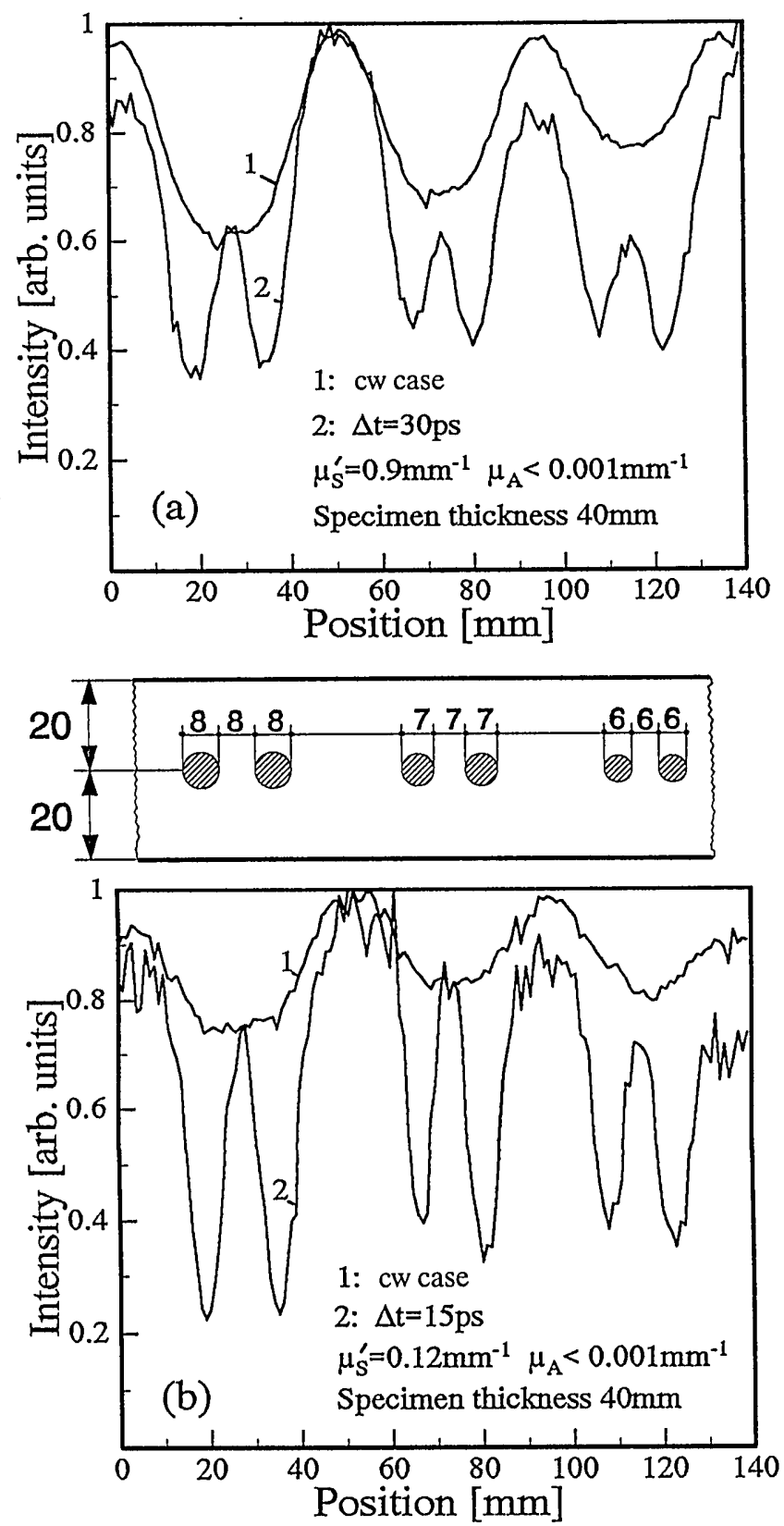

Fig. 8. Line scan across pairs of blackened beads with diameters of 6,7 , and $8 \mathrm{~mm}$. Reduced scattering coefficients of the surrounding medium: (a) $\mu_{s}{ }^{\prime}=0.9 \mathrm{~mm}^{-1}$, (b) $\mu_{s}{ }^{\prime}=0.12 \mathrm{~mm}^{-1}$. sity (Fig. 5). The light intensity is normalized to the $\mathrm{cw}$ case and is shown on a logarithmic scale. It becomes evident that with a shorter time gate, any gain of spatial resolution must be paid for by a very fast reduction of signal light intensity.

In Fig. 6 the influence of increasing absorption is investigated. The absorption coefficient is increased from $\mu_{A}<0.001 \mathrm{~mm}^{-1}$ to $\mu_{A}=0.012 \mathrm{~mm}^{-1}$ by the addition of ink. As seen in Fig. 2, the increased absorption reduces the number of scattered photons at late times. Consequently the spatial resolution is improved even in the cw case (last symbol on each curve) ${ }^{24}$ For short gate times the improvement is
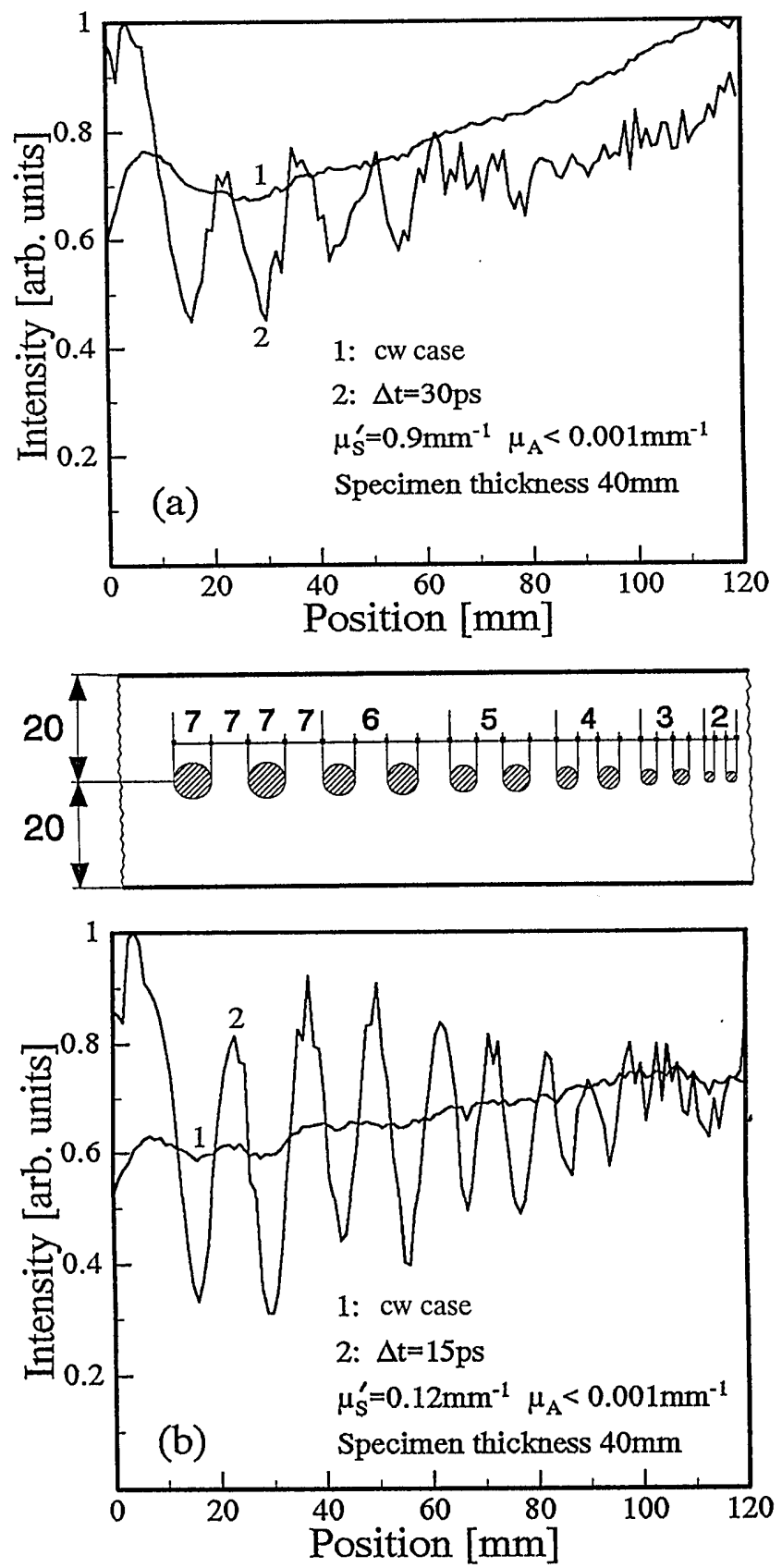

Fig. 9. Line scan across a chain of blackened beads with diameters of $2-7 \mathrm{~mm}$. Reduced scattering coefficients of the surrounding medium: (a) $\mu_{s}{ }^{\prime}=0.9 \mathrm{~mm}^{-1}$, (b) $\mu_{s}{ }^{\prime}=0.12 \mathrm{~mm}^{-1}$. 
not evident. The overall benefit of time gating becomes smaller, and the resolution gain factor is reduced from 1.9 (Fig. 6, curve 1) to 1.6 (Fig. 6, curve 2).

Complementary measurements on phantoms with different reduced scattering coefficients were performed to study the influence of $\mu_{s}{ }^{\prime}$ on the improvement achievable in spatial resolution by time gating. Figure 7 shows the spatial resolution as a function of the end-of-gate time $t_{E}$ for reduced scattering coefficients of (a) $\mu_{s}{ }^{\prime}=0.12-4.5 \mathrm{~mm}^{-1}$ and (b) $\mu_{s}{ }^{\prime}=$ $0.048-0.12 \mathrm{~mm}^{-1}$. The spatial resolution for the $\mathrm{cw}$ case (last symbol on each curve) becomes worse with a decreasing reduced scattering coefficient up to $\mu_{s}{ }^{\prime}=$ $0.12 \mathrm{~mm}^{-1}$ [Fig. 7(a)]. This behavior is due to scattering and not to absorption and has also been verified by Monte Carlo simulations. ${ }^{25}$ If the reduced scattering coefficient is decreased further $\left(\mu_{s}{ }^{\prime}<0.12 \mathrm{~mm}^{-1}\right)$, the turbid medium gradually becomes transparent and the resolution for the cw case is again improved [Fig. 7(b)]. Time-gated detection is especially advantageous when the spatial resolution of the cw case is lowest, i.e., near $\mu_{s}{ }^{\prime}=0.12 \mathrm{~mm}^{-1}$ under the present condition of low absorption $\mu_{A}<0.001 \mathrm{~mm}^{-1}$ and a specimen thickness of $40 \mathrm{~mm}$. The resolution gain factor attainable by a time-gating method rises from 1.6 at $\mu_{s}{ }^{\prime}=4.5 \mathrm{~mm}^{-1}$ to 2.8 for $\mu_{s}{ }^{\prime}=0.12 \mathrm{~mm}^{-1}$. If $\mu_{s}{ }^{\prime}$ is decreased further, the benefit of time gating becomes smaller. For typical optical parameters of the human breast tissue of Table 1, one expects an improvement of the spatial resolution, which is due to time-gated detection, by a factor of 2 .
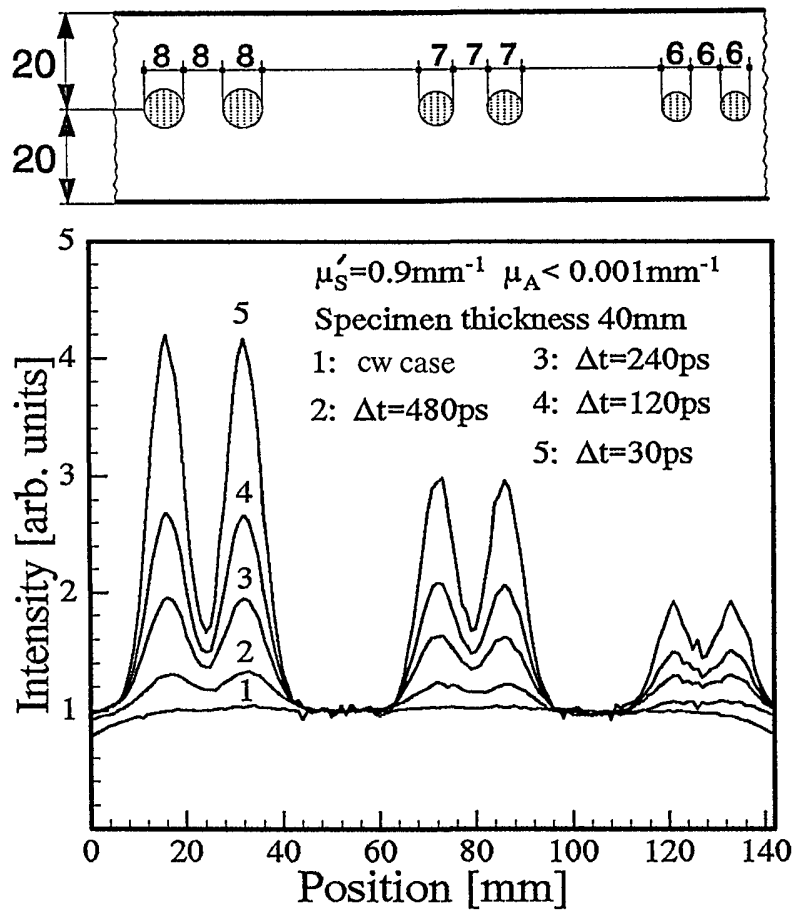

Fig. 10. Scan across bead pairs from Plexiglas with bead diameters of 6,7 , and $8 \mathrm{~mm}$.

\section{B. Detectability of Completely Absorbing Objects}

To corroborate the statements of Subsection 4.A, measurements were also performed on bead pairs to determine the physical resolution in a more realistic case. Pairs of blackened beads with a separation equal to their diameter were placed in the central plane of the scattering medium. The measurements were performed on phantoms with $\mu_{s}^{\prime}=0.9 \mathrm{~mm}^{-1}$ [Fig. 8(a)] and $\mu_{s}{ }^{\prime}=0.12 \mathrm{~mm}^{-1}[$ Fig. $8(\mathrm{~b})]$ and bead diameters of 6,7 , and $8 \mathrm{~mm}$. In the cw case the individual beads cannot be resolved at either the large
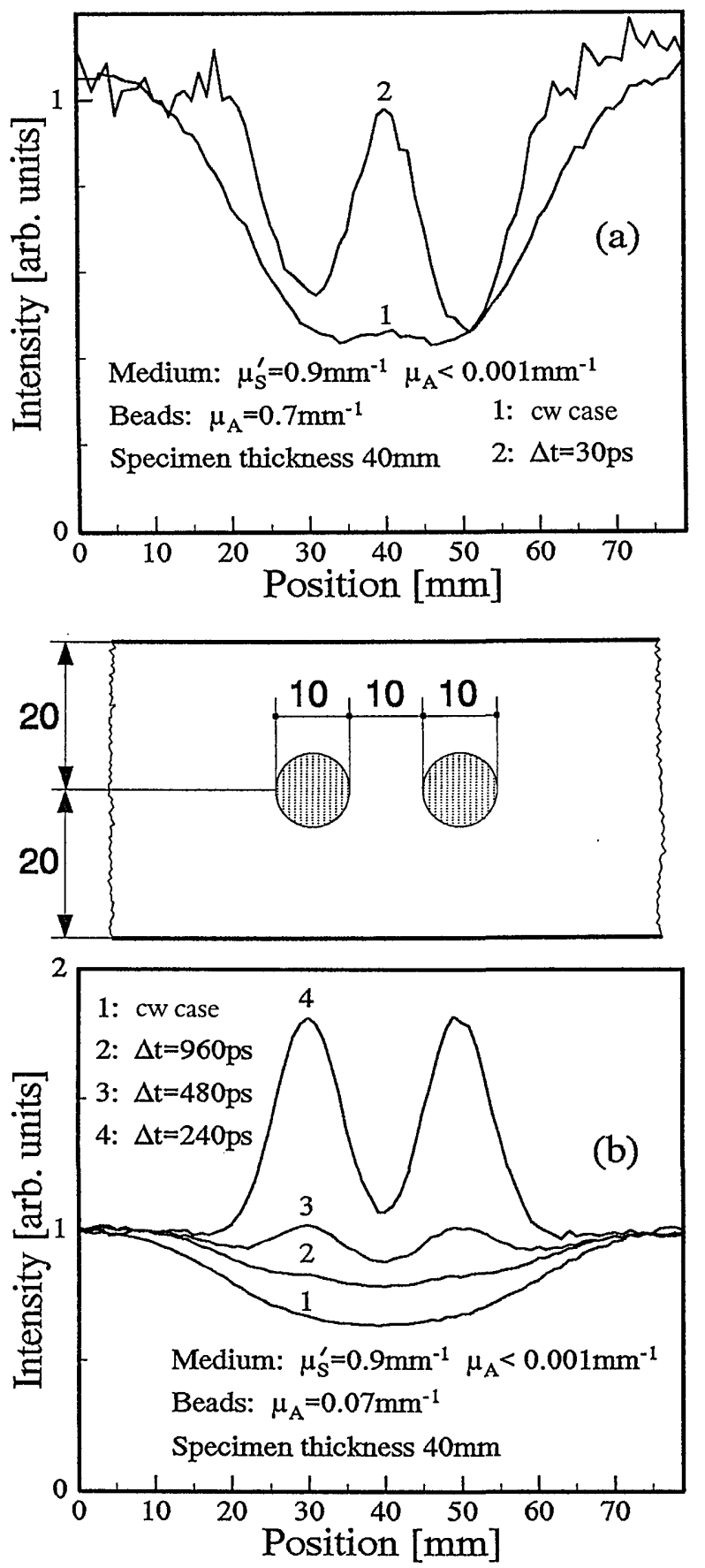

Fig. 11. Scan across bead pairs from partially absorbing plastic with absorption coefficients (a) $\mu_{A}=0.7 \mathrm{~mm}^{-1}$, (b) $\mu_{A}=0.07 \mathrm{~mm}^{-1}$. 

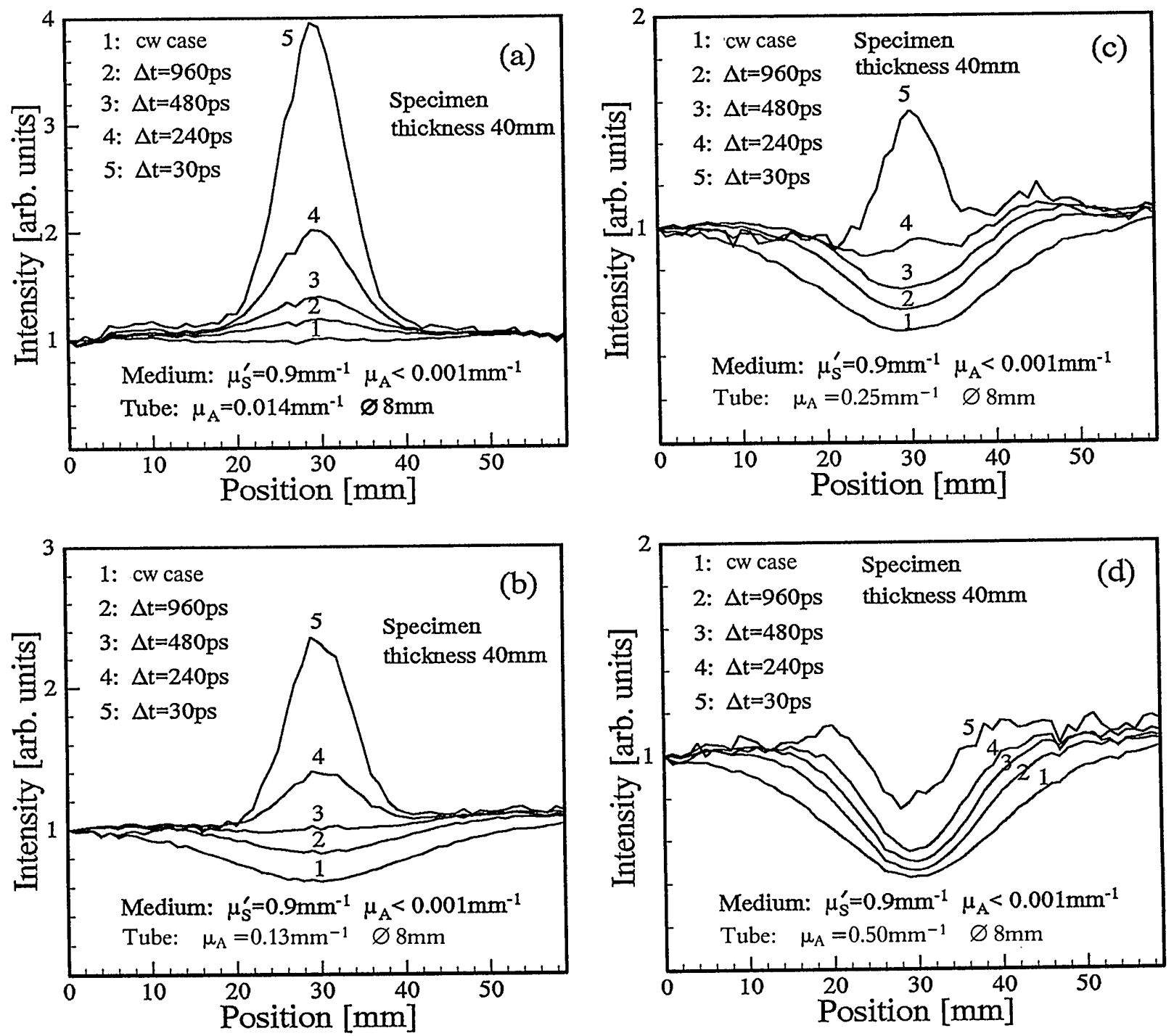

Fig. 12. Scan across plastic tubes filled with diluted ink. Corresponding absorption coefficients: (a) $\mu_{A}=0.014 \mathrm{~mm}^{-1},(\mathrm{~b}) \mu_{A}=0.13$ $\mathrm{mm}^{-1}$, (c) $\mu_{A}=0.25 \mathrm{~mm}^{-1}$, (d) $\mu_{A}=0.50 \mathrm{~mm}^{-1}$.

or the small reduced scattering coefficient. At the larger reduced scattering coefficient, however, the individual pairs can be seen with higher contrast than in the case of a smaller one. The time gate for the measurement with $\mu_{s}{ }^{\prime}=0.9 \mathrm{~mm}^{-1}$ has a value of $\Delta t=$ 30 ps. A still shorter integration time hardly improves the spatial resolution, but merely impairs the signal-to-noise ratio. For $\mu_{s}{ }^{\prime}=0.12 \mathrm{~mm}^{-1}$ with a time gate of $\Delta t=15 \mathrm{ps}$, the performance limit of the time-resolving system is gradually reached. The time gate starts at the times of flight of 350 ps [Fig. 8(a)] and 180 ps [Fig. $8(\mathrm{~b})$ ], where the signal overcomes the background noise. With time gating, even the 6-mm beads can be distinguished in both cases and more clearly for smaller reduced scattering coefficients than for larger ones. In Fig. 9 measurements for (a) $\mu_{s}^{\prime}=0.9 \mathrm{~mm}^{-1}$ and (b) $\mu_{s}^{\prime}=0.12 \mathrm{~mm}^{-1}$ with $\mu_{A}<$ $0.001 \mathrm{~mm}^{-1}$ on a phantom consisting of a bead chain with diameters of 2 to $7 \mathrm{~mm}$ are shown for completeness. For $\mu_{s}^{\prime}=0.12 \mathrm{~mm}^{-1}$ with a time gate of $\Delta t=$
$15 \mathrm{ps}$, the 4 to $7-\mathrm{mm}$ beads can be seen as separated [Fig. 9(b)], whereas even the 7-mm beads are not resolved in the $\mathrm{cw}$ case. A measurement with a greater reduced scattering coefficient $\mu_{s}{ }^{\prime}=0.9 \mathrm{~mm}^{-1}$ [Fig. 9(a)] shows that beads with diameters $\geq 6 \mathrm{~mm}$ are distinguished in the time-resolved detection.

\section{Detectability of Partially Absorbing Objects}

The detection of completely absorbing objects in the surrounding scattering medium represents a special case that is of limited importance for practical applications. To demonstrate the sensitivity of the timeresolving technique for objects encountered in medical diagnostics, measurements were performed on partially absorbing objects that differ from their environment in terms of scattering and absorption. ${ }^{26,27}$ Figure 10 shows a line scan over pairs of beads from Plexiglas with diameters of 6,7 , and $8 \mathrm{~mm}$ in a turbid medium with $\mu_{s}{ }^{\prime}=0.9 \mathrm{~mm}^{-1}$ and $\mu_{A}<0.001 \mathrm{~mm}^{-1}$. The surfaces of the beads have been polished and are 

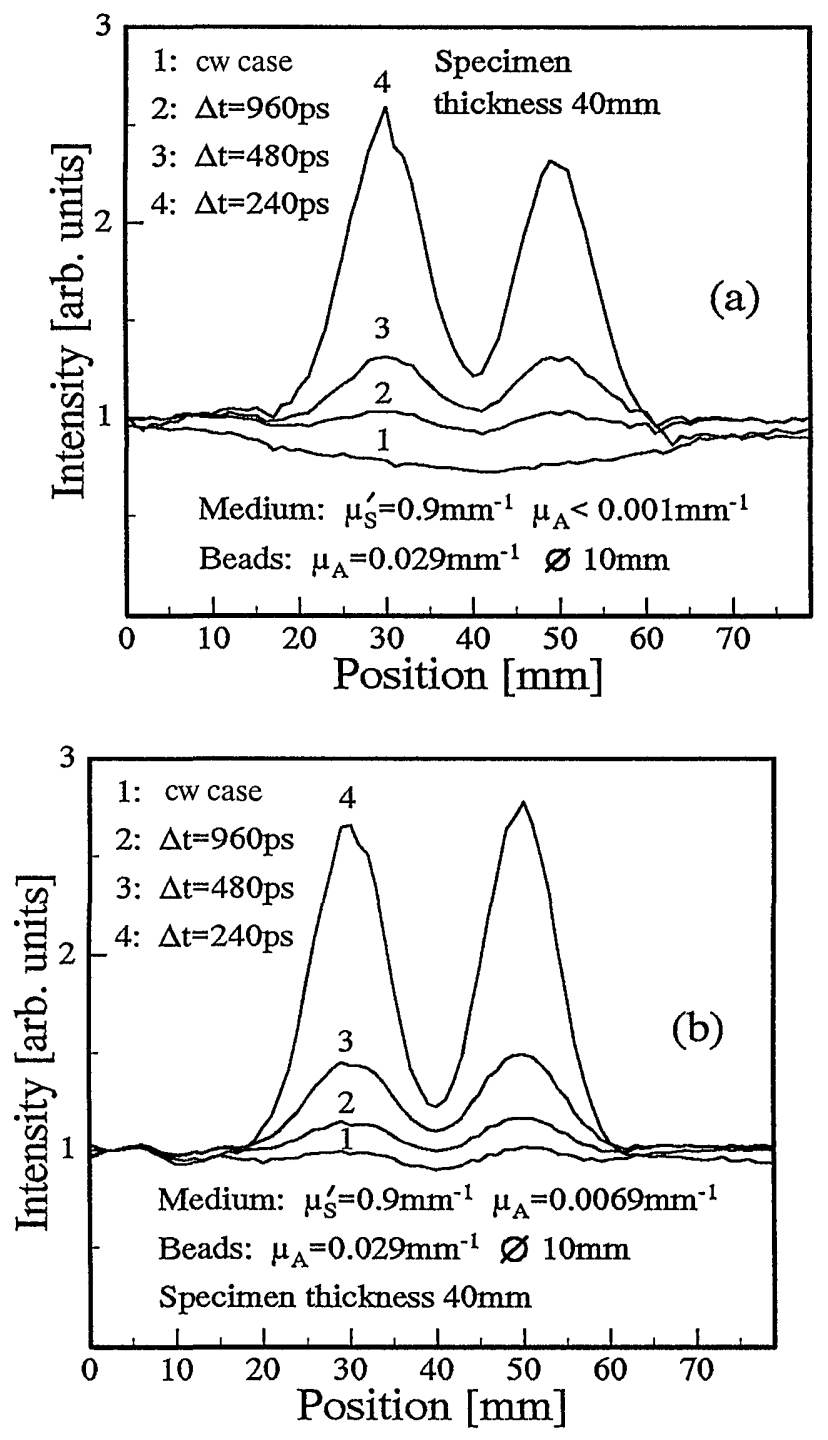

therefore smooth and clear. With a short integration time of $\Delta t=30 \mathrm{ps}$, the $6-\mathrm{mm}$ bead pair can be resolved. In the cw case, however, not even the pairs themselves can be detected in contrast to the case in which the bead pairs were blackened (Fig. 8). Apparently the improvement of resolution originates from the fact that the path length of photons that traverse the Plexiglas bead pair is shorter. Therefore the absorption of the beads is of special importance. Figure 11 shows line scans over bead pairs with bead diameters of $10 \mathrm{~mm}$. The absorption in the beads is $\mu_{A}=0.7 \mathrm{~mm}^{-1}$ [Fig. 11(a)] or $\mu_{A}=0.07$ $\mathrm{mm}^{-1}$ [Fig. 11(b)] with negligible scattering. The line scan of the strongly absorbing beads [Fig. 11(a)] yields results that are analogous to those of the blackened beads (see Fig. 8), i.e., a signal reduction can be observed in cases of both time-gated and cw detection. With reduced absorption within the beads [Fig. 11(b)], signal shape and amplitude depend strongly on the time gate $\Delta t$. For cw detection and longer time gates of $\Delta t>480 \mathrm{ps}$ a signal reduction is observed, whereas for shorter time gates of $\Delta t<480$

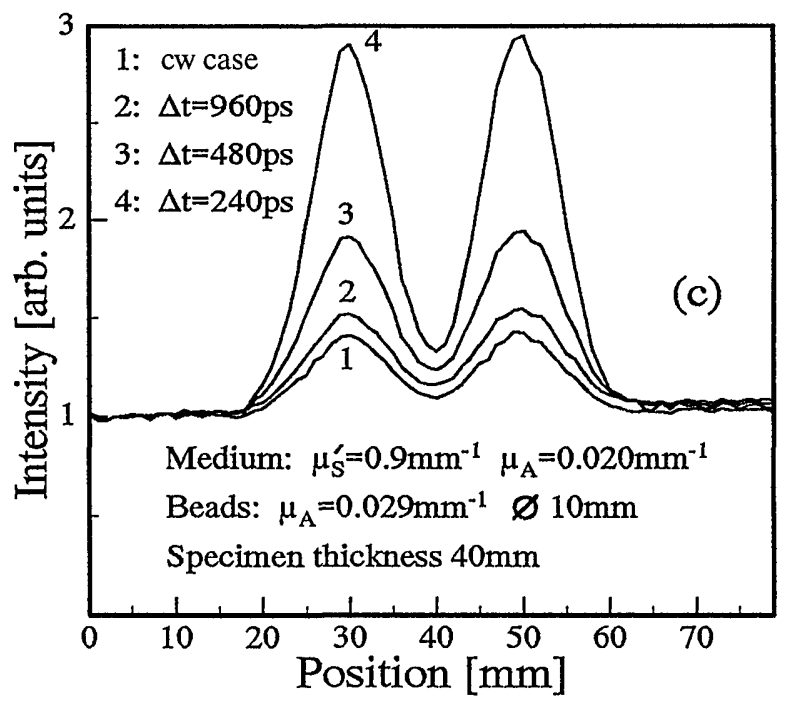

Fig. 13. Scan across bead pairs from partially absorbing plastic in surrounding media with absorption coefficients (a) $\mu_{A}<0.001$ $\mathrm{mm}^{-1}$, (b) $\mu_{A}=0.0069 \mathrm{~mm}^{-1}$, (c) $\mu_{A}=0.020 \mathrm{~mm}^{-1}$.

ps the signal is increased, and a strong improvement in resolution occurs. The beads in the scattering medium appear to be more transparent with decreasing integration time.

For examining this observation in more detail, measurements were made on plastic tubes $(8-\mathrm{mm}$ diameter) filled with successively diluted ink. Figure 12 shows a series of measurements in which the absorption coefficient in the tubes was changed from $\mu_{A}=0.014 \mathrm{~mm}^{-1}$ to $\mu_{A}=0.50 \mathrm{~mm}^{-1}$. Scattering within the tubes was negligible. The result of Fig. 12 (a) with $\mu_{A}=0.014 \mathrm{~mm}^{-1}$ is analogous to the measurements on Plexiglas beads, i.e., the tube is transparent. If the absorption coefficient increases to $\mu_{A}=0.13 \mathrm{~mm}^{-1}$ [Fig. $12(\mathrm{~b})$ ], a signal increase is observed for integration times shorter than $\Delta t=480$ ps, whereas the signal is reduced for longer integration times up to the $\mathrm{cw}$ case. If the absorption coefficient in the plastic tube is raised further to $\mu_{A}=$ $0.25 \mathrm{~mm}^{-1}$ [Fig. 12(c)], then the integration time at which the conditions tip over to a signal increase is even shorter. If the absorption coefficient attains 


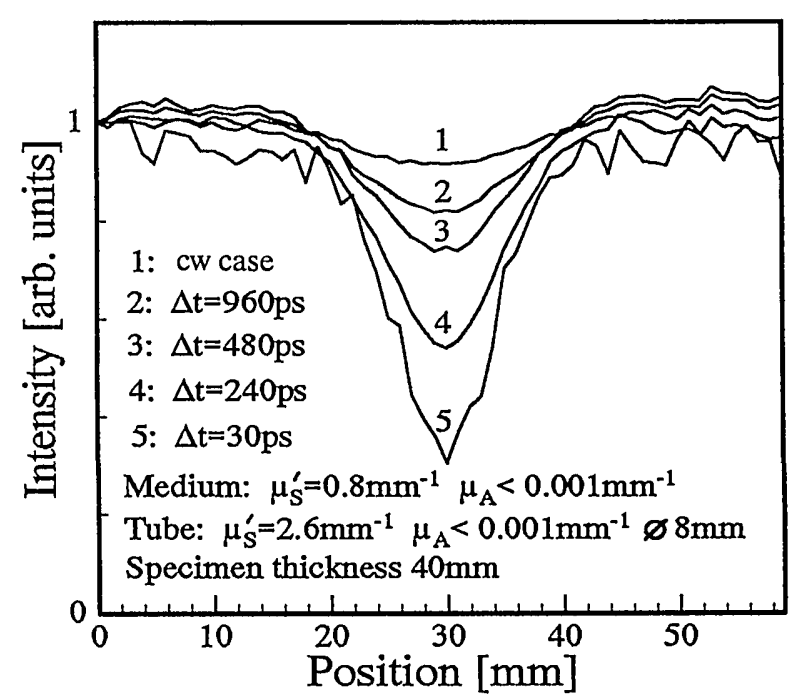

Fig. 14. Scan across a plastic tube filled with condensed milk, which differs only in terms of scattering from the surrounding medium.

values of $\mu_{A}=0.50 \mathrm{~mm}^{-1}$ [Fig. 12(d)], then the tube appears to be absorbing in all cases, even at the shortest integration time of $\Delta t=30 \mathrm{ps}$. This behavior is understandable, as photons from the surrounding scattering medium traverse the plastic tube several times because of the low scattering there and the high scattering of the surrounding medium. Because of the absorption occurring in the tube and depending on the number of traverses, either an increase or a reduction of the signal is obtained. The number of traverses depends on the respective time gate. The smaller the time gate, the less frequently the tubes are traversed by the corresponding photons. This explains phenomenologically why the tip-over integration time becomes successively shorter with increasing absorption within the plastic tubes.

All experiments shown above were made with negligible light absorption in the scattering medium. In general, increasing absorption within the medium improves the spatial resolution in the $\mathrm{cw}$ case, and time gating is less advantageous (Fig. 6). Figure 13 shows a series of measurements with gradually increasing absorption coefficients $\mu_{A}$ of the surrounding medium. A pair of beads with $\mu_{A}=0.029 \mathrm{~mm}^{-1}$ and negligible scattering similar to the measurement of Fig. 11(b) has been used (10-mm diameter). In Fig. 13(a) the turbid medium has a reduced scattering coefficient $\mu_{s}{ }^{\prime}=0.9 \mathrm{~mm}^{-1}$ and negligible absorption. As the absorption coefficient within the beads is smaller than that of the beads in Fig. 11(b), the signal reduction in the cw case is not as large as in Fig. 11(b). If the absorption coefficient in the medium increases to $\mu_{A}=0.0069 \mathrm{~mm}^{-1}$ [Fig. 13 (b)] the signal reduction at the bead position of the cw case practically vanishes. For $\mu_{A}=0.020 \mathrm{~mm}^{-1}$ in the scattering medium [Fig. 13(c)] the beads can be distinguished even in the $\mathrm{cw}$ case. Because of the increasing absorption coefficient in the surrounding medium, the signal reduction at the bead position has changed into a signal

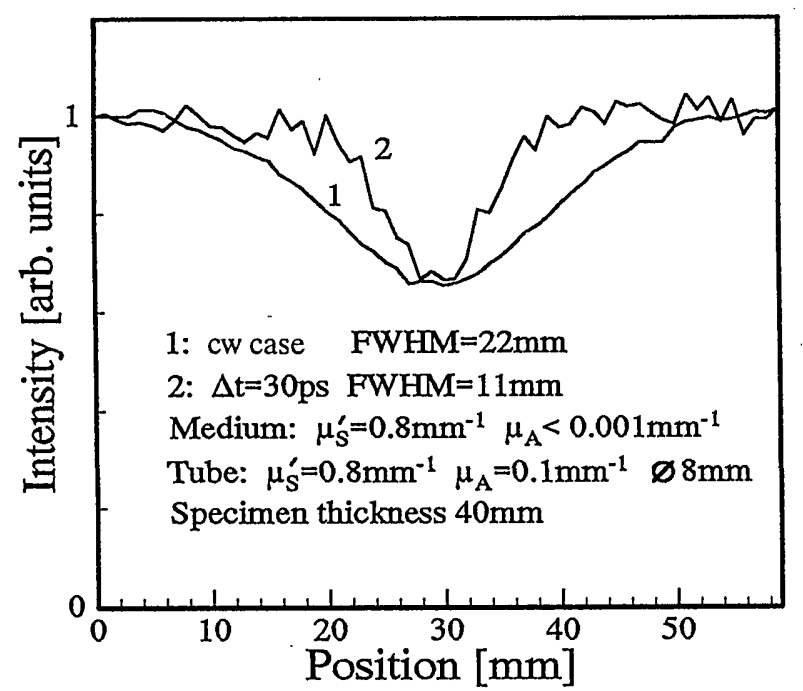

Fig. 15. Scan across a plastic tube filled with ink-tinted milk, which differs only in terms of absorption from the surrounding medium.

increase for the cw detection and longer time gates. For shorter time gates of $\Delta t \approx 240 \mathrm{ps}$, the influence of the increased absorption coefficient is hardly noticeable because of the shorter optical path length of the corresponding photons. The use of time gating improves the spatial resolution but is not as beneficial as in the case of negligible absorption of the turbid medium.

Figure 14 shows a line scan across a plastic tube filled with condensed milk. The surrounding turbid medium has a reduced scattering coefficient $\mu_{s}{ }^{\prime}=0.8$ $\mathrm{mm}^{-1}$, whereas for the tube $\mu_{s}^{\prime}=2.6 \mathrm{~mm}^{-1}$ (absorption coefficient $\mu_{A}<0.001 \mathrm{~mm}^{-1}$ ). With a time gate of $\Delta t=30 \mathrm{ps}$, the tube can be detected as a significant signal reduction, whereas the cw case permits hardly any detection. Finally, Fig. 15 shows an object with the same scattering but higher absorption than the surrounding medium. The tube with ink-tinted milk, imaged with a time gate of $\Delta t=30 \mathrm{ps}$, is resolved with better spatial resolution than for the cw detection. The full width at half-maximum (FWHM) is reduced from $22 \mathrm{~mm}$ for the cw detection to $11 \mathrm{~mm}$ for a time gate of $\Delta t=30 \mathrm{ps}$. However, the contrast is not improved. If this result is compared with that of Fig. 14, it can be seen that time gating improves the detection of inhomogeneities if they differ from their environment in terms of scattering. ${ }^{28}$

\section{Summary}

The optical parameters of the female breast were determined by in vivo experiments at a wavelength of $\lambda=800 \mathrm{~nm}$ (Table 1). Systematic phantom experiments with a wide range of reduced scattering and absorption coefficients for both the highly scattering medium and embedded objects have been performed. The variety of different embedded objects permits the estimation of the benefit of time gating for breast imaging. 
The phantom experiments described here show that the use of time-resolving techniques in the transillumination of turbid media offers a clear gain in spatial resolution. For completely absorbing objects, this gain depends on the optical parameters of the surrounding medium, shown by measurements on blackened beads (Subsection 4.B). For comparison of the spatial resolution achieved for $\mathrm{cw}$ illumination with that achieved for time gating, a gain factor calculated from edge-spread functions has been introduced. For a specimen thickness of $40 \mathrm{~mm}$ the greatest benefit from time gating is obtained for comparatively small reduced scattering coefficients near $\mu_{s}{ }^{\prime}=0.12 \mathrm{~mm}^{-1}$, which unfortunately represent a minority of biological applications. With an increasingly reduced scattering coefficient $\mu_{s}{ }^{\prime}>0.12 \mathrm{~mm}^{-1}$, the gain in spatial resolution is reduced. The gain factor, determined from edge-spread functions, is 1.6 for $\mu_{s}{ }^{\prime}=4.5 \mathrm{~mm}^{-1}, 1.9$ for $\mu_{s}{ }^{\prime}=0.9 \mathrm{~mm}^{-1}$, and 2.8 for $\mu_{s}{ }^{\prime}=0.12 \mathrm{~mm}^{-1}$. With increasing specimen thicknesses these gain factors are decreased, and the reduced scattering coefficient for which the maximum gain factor is achieved shifts to smaller values.

With time-resolved detection, completely absorbing bead pairs with a diameter of $6 \mathrm{~mm}$ can be distinguished for a turbid medium with $\mu_{s}{ }^{\prime}=0.9 \mathrm{~mm}^{-1}$, whereas 4-mm bead pairs can be separated at $\mu_{s}{ }^{\prime}=$ $0.12 \mathrm{~mm}^{-1}$ (specimen thickness $40 \mathrm{~mm}$ ). For the cw detection not even a bead pair with a diameter of 8 $\mathrm{mm}$ can be separated in both cases, the large and the small reduced scattering coefficients.

Increasing absorption within the scattering medium reduces the benefit of time gating. For highly absorbing scattering media, the use of time-resolved detection is not profitable. If the immersed objects are only partially absorbing, the measurements show that the time resolution is especially advantageous. In this case time-resolved experiments should permit obtaining information on the relative optical properties of the inhomogeneity and the surrounding medium.

The conclusion is that the spatial resolution of time-gated breast imaging is not considerably better than $10 \mathrm{~mm}$. It is impossible to detect photons at very short times of flight (the so-called ballistic and snake photons) and therefore femtosecond pulses and ultrafast shutters are not useful for time-gated breast imaging. For finally deciding what practical benefit time gating offers, further in vivo experiments are necessary. Nevertheless, the question of how to realize time gating for practical applications is still unclear. At the present less than 1\% of transmitted light can be used for time-resolved imaging, and long measurement times restrict practical applications. However, different approaches have been published to overcome this limitation. ${ }^{7,29}$

We thank A. Oppelt and his co-workers, especially $K$. Klingenbeck-Regn and O. Schütz, for their support and cooperation, and $\mathrm{H}$. Bartelt and his colleagues for helpful discussions. Special thanks are due to $M$. Guntersdorfer and E. Wolfgang for their encourage- ment and continual support. We are obliged to $\mathrm{C}$. Hauger, I. Jung, and T. Wilhelm for their technical support regarding the Ti:sapphire laser. We gratefully acknowledge physicians A. Dollinger, S. H. Heywang-Köbrunner, and A. Schlegel for important assistance during the in vivo experiments.

\section{References}

1. O. Jarlman, G. Balldin, I. Andersson, M. Löfgren, A. S. Larsson, and F. Linell, "Relation between lightscanning and the histologic and mammographic appearance of malignant breast tumors," Acta Radiol. 33, 63-68 (1992).

2. O. Jarlman, I. Andersson, G. Balldin, and S. A. Larsson, "Diagnostic accuracy of light scanning and mammography in women with dense breast," Acta Radiol. 33, 69-71 (1992).

3. B. Chance, S. Nioka, J. Kent, K. McCully, M. Fountain, R. Greenfeld, and G. Holtom, "Time-resolved spectroscopy of haemoglobin and myoglobin in resting and ischemic muscle," Anal. Biochem. 174, 698-707 (1988).

4. B. Chance, J. S. Leigh, H. Miyake, D. S. Smith, S. Nioka, R. Greenfeld, M. Finander, K. Kaufmann, W. Levy, M. Young, P. Cohen, H. Yoshioka, and R. Boretsky, "Comparison of timeresolved and -unresolved measurements of deoxyhemoglobin in the brain," Proc. Natl. Acad. Sci. U.S.A. 85, 4971-4975 (1988).

5. D. T. Delpy, M. Cope, P. van der Zee, S. Arridge, S. Wray, and J. Wyatt, "Estimation of optical path length through tissue from direct time-of-flight measurement," Phys. Med. Biol. 33, 1422-1433 (1988).

6. J. R. Lakowicz and K. Berndt, "Frequency-domain measurements of photon migration in tissues," Chem. Phys. Lett. 166(3), 246-252 (1990).

7. T. French, E. Gratton, and J. Maier, "Frequency domain imaging of thick tissues using a CCD," in Time-Resolved Laser Spectroscopy in Biochemistry III, J. R. Lakowicz, ed., Proc. Soc. Photo-Opt. Instrum. Eng. 1640, 254-261 (1992).

8. A. Knüttel, J. M. Schmitt, and J. R. Knutson, "Improvement of spatial resolution in reflectance near-infrared imaging by laser-beam interference," in Time-Resolved Laser Spectroscopy in Biochemistry III, J. R. Lakowicz, ed., Proc. Soc. Photo-Opt. Instrum. Eng. 1640, 405-416 (1992).

9. S. L. Jacques, "Principles of phase-resolved optical measurements," in Future Trends in Biomedical Applications of Lasers, L. O. Svaasand, ed., Proc. Soc. Photo-Opt. Instrum. Eng. 1525, 143-153 (1991).

10. J. C. Hebden, R. A. Kruger, and K. S. Wong, "Time resolved imaging through a highly scattering medium," Appl. Opt. 30, 788-794 (1991).

11. J. C. Hebden and R. A. Kruger, "Transillumination imaging performance: a time-of-flight imaging system," Med. Phys. 17, 351-356 (1990).

12. R. Berg, S. Andersson-Engels, O. Jarlman, and S. Svanberg, "Tumor detection using time-resolved light transillumination," in Future Trends in Biomedical Applications of Lasers, L. O. Svaasand, ed., Proc. Soc. Photo-Opt. Instrum. Eng. 1525, 59-67 (1991).

13. G. Mitic, J. Kölzer, J. Otto, and E. Plies, "Zeitaufgelöste Transillumination von trüben Medien," in Lasers in Medicine, W. Waidelich, and A. Hofstetter, eds. (Springer-Verlag, Berlin, 1993), pp. 479-484.

14. R. Berg, O. Jarlman, and S. Svanberg, "Medical transillumination imaging using short-pulse diode lasers," Appl. Opt. 32, 574-579 (1993).

15. S. Andersson-Engels, R. Berg, and S. Svanberg, "Timeresolved transillumination for medical diagnostics," Opt. Lett. 15, 1179-1181 (1990).

16. W. F. Cheong, S. A. Prahl, and A. J. Welch, "A review of the 
optical properties of biological tissues," IEEE J. Quantum Electron. 26, 2166-2184 (1990).

17. M. S. Patterson, B. Chance, and B. C. Wilson, "Time-resolved reflectance and transmittance for the noninvasive measurements of optical properties," Appl. Opt. 28, 2331-2336 (1989).

18. R. Berg, S. Andersson-Engels, O. Jarlman, and S. Svanberg, "Time-resolved transillumination for medical diagnostics," in Time-Resolved Spectroscopy and Imaging of Tissues, B. Chance and A. Katzir, eds., Proc. Soc. Photo-Opt. Instrum. Eng. 1431, 110-119 (1991).

19. Radiation Safety of Laser Products, Equipment Classification Requirements and User's Guide (International Electrotechnical Commission, 1990).

20. H. Key, E. R. Davies, P. C. Jackson, and P. N. T. Wells, "Optical attenuation characteristics of breast tissues at visible and near-infrared wavelengths," Phys. Med. Biol. 36, 579-590 (1991).

21. V. G. Peters, D. R. Wyman, M. S. Patterson, and G. L. Frank, "Optical properties of normal and diseased human breast tissues in the visible and near infrared," Phys. Med. Biol. 35, 1317-1334 (1990).

22. J. C. Hebden, "Evaluating the spatial resolution performance of a time-resolved optical imaging system," Med. Phys. 19, 1081-1087 (1992).

23. J. B. Fishkin and E. Gratton, "Diffraction of intensity modulated light in strongly scattering media in the presence of a "semi-infinite" absorbing or reflecting plane bounded by a straight edge," in Time-Resolved Laser Spectroscopy in Biochemistry III, J. R. Lakowicz, ed., Proc. Soc. Photo-Opt. Instrum. Eng. 1640, 362-367 (1992).
24. O. Schuetz, H. E. Reinfelder, K. Klingenbeck-Regn, and H. Bartelt, "Monte Carlo modelling of time-resolved nearinfrared transillumination of human breast tissue," in Laser Light Scattering in Medical Diagnostics and Therapy, B. Chance, D. T. Delpy, M. Ferrari, M. J. van Gemert, G. J. Mueller, and V. V. Tuchin, eds., Proc. Soc. Photo-Opt. Instrum. Eng. 2082, 123-129 (1993).

25. F. Spiegel and H. Pulvermacher, "Optical transfer function and resolution of transillumination processes calculated by Monte Carlo simulation and diffusion theory," in Laser Light Scattering in Medical Diagnostics and Therapy, B. Chance, D. T. Delpy, M. Ferrari, M. J. van Gemert, G. J. Mueller, and V. V. Tuchin, eds., Proc. Soc. Photo-Opt. Instrum. Eng. 2082, 86-97 (1993).

26. J. C. Hebden, "Time-resolved imaging of opaque and transparent spheres embedded in a highly scattering medium," Appl. Opt. 32, 3837-3841 (1993).

27. G. Mitic, J. Kölzer, J. Otto, E. Plies, G. Sölkner, and W. Zinth, "Time-resolved transillumination of turbid media," in Laser Light Scattering in Medical Diagnostics and Therapy, B. Chance, D. T. Delpy, M. Ferrari, M. J. van Gemert, G. J. Mueller, and V. V. Tuchin, eds., Proc. Soc. Photo-Opt. Instrum. Eng. 2082, 26-32 (1993).

28. S. Andersson-Engels, R. Berg, and S. Svanberg, "Effects of optical constants on time-gated transillumination of tissue and tissue-like media," J. Photochem. Photobiol. B Biol. 16, 155-167 (1992).

29. J. C. Hebden, "Line scan acquisition for time-resolved imaging through scattering media," Opt. Eng. 32, 626-633 (1993). 